\title{
Pflege und emotionale Verantwortung
}

\author{
So nah am Patienten zu sein, bedeutet auch große Nähe der Gefühle
}

Gefühle spielen im Zusammenhang mit Krankheit eine wichtige Rolle - und werden doch im Gesamtgeschehen der Behandlung oft nicht entsprechend wahrgenommen und berücksichtigt. Beim 2. LEBENS.MEDinzinischen Kongress Pflege Anfang Juni in Bad Erlach in Südniederösterreich standen sie unter dem Titel „Das Leben spüren... - Emotionen wahrnehmen" im Fokus von Interviews, Workshops und Vorträgen. Wichtig ist, im Pflegebereich emotionale Verantwortung zu übernehmen“, erklärte der Pflegedienstleiter des Lebens.Med Zentrums Bad Erlach, DGKP Jürgen Friedl.

Die Erwartungen, Probleme und Umsetzung in der Kommunikation und Verarbeitung von „schlechten“ Nachrichten, wie sie die Diagnose Krebs darstellt, erläuterten: Ein Krebspatient, eine Diplomierte Pflegekraft und ein Arzt. „Ein solches Gespräch muss immer gut vorbereitet sein und es ist jede Menge Empathie gefragt", betonten Univ.-Prof. Dr. Alexander Gaiger, Ärztlicher Leiter, Onkologische Rehabilitation am Lebens.Med Zentrum, und DGKS Elisabeth Seiler, Onkologie am Landesklinikum Wiener Neustadt. Der Einblick in das private Tagebuch von Krebspatient Dr. Karl H. Exinger zeigte die persönlichen Höhen und Tiefen. Als Lösungsansatz, der in speziellen Workshops herausgearbeitet wurde, zeigte sich die Bedeutung der persönlichen Grundstimmung des Betroffenen. Die individuelle Vorgehensweise muss sich daran orientieren - und auch Humor kann durchaus angebracht sein, wie DGKP Jörg Fuhrmann, MSc., Hausund Pflegedienstleiter im Seniorenwohnhaus Großarl, Seminarleiter und Referent feststellte. Neben dem Patienten ist meist auch sein Umfeld von der Krebserkrankung sehr stark emotional betroffen: Wie Kinder mit der Diagnose Krebs bei ihren Eltern umgehen sowie Hilfestellungen dazu, behandelte die Klinische und Gesundheitspsychologin Mag. Jutta Steinschaden von der Krebshilfe Wien und hob dabei besonders die Initiative „Rehabilitation mit Kind" des Lebens.Med Zentrums Bad Erlach hervor. Zu bestimmten Turnussen können Eltern ihren Rehabilitationsaufenthalt mit ihrem Kind gestalten, dafür wird eine Betreuung für das Kind

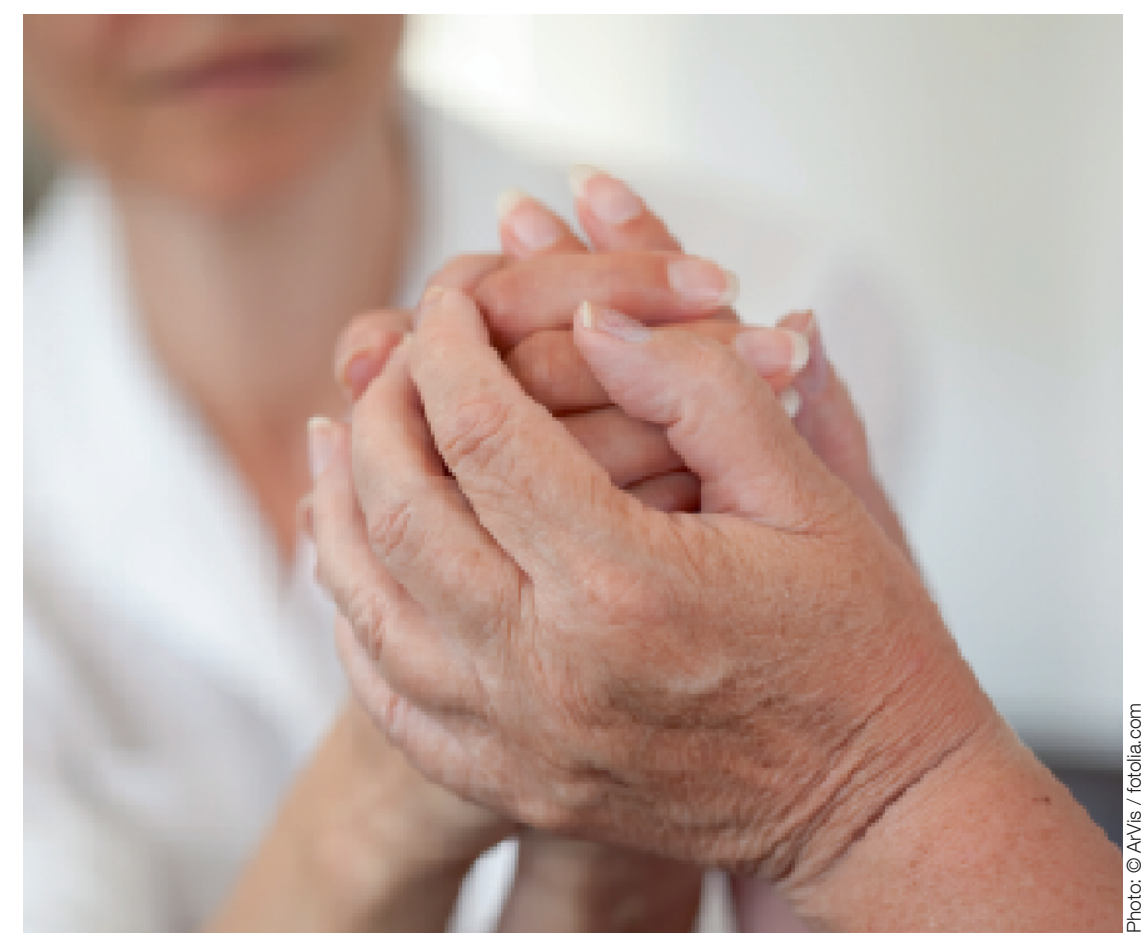

während der Therapiezeit direkt im Lebens.Med Zentrum Bad Erlach geboten.

\section{Selbstbild und \\ Selbstwahrnehmung}

Von großen Emotionen sind beispielsweise auch Narben im Allgemeinen nach großen operativen Eingriffen oder Unfällen, und ganz speziell nach Brustkrebsoperationen begleitet, wie Univ.-Prof. Mag. Dr. Hanna Mayer, Institut für Pflegewissenschaft, Universität Wien feststellte. Anhand praktischer Beispiele und wissenschaftlicher Erkenntnisse verdeutlichte sie dies in Bezug auf Selbstwahrnehmung und Selbstbild als zentralen Konzepten des Erlebens von Frauen mit Brustkrebs.

Die Pflege als Berufsgruppe, die so nah am Patienten ist, wie sonst keiner, ist immer wieder mit dem Thema Berührung konfrontiert: Berührung im Rahmen von Pflegehandlungen, Berührung aber auch als gegenseitiger Patientenkontakt. Berührungen haben in der Pflege viele Bedeutungen, wie DGKS Doris Kamleitner, BScN, MA, Unfallkrankenhaus Wien Lorenz Böhler berichtete und auch die Grenzen im Hinblick auf Berührungen aufzeigte.
Hochleistung im Sport und in der Pflege

Zwischen Sport und dem Pflegeberuf lassen sich übrigens durchaus ähnliche Setting-Charakteristika beobachten - beide Tätigkeitsfelder finden im Hochleistungsbereich statt: „Euphorie, Motivation aber auch Niederlage und Stagnation sind Faktoren, welche zentrale Punkte in beiden Bereichen darstellen“, beschrieb Mag. Andreas Haller, Bakk., Wissenschaftlicher Mitarbeiter Fachhochschule Wiener Neustadt, Lehrgang Training und Sport.

„Pflege ohne Emotionen ist keine Pflege“, resümierte Fuhrmann: „Man bekommt unendlich viel zurück, wenn man sich öffnet." Und forderte die Pflegekräfte auf: „Spüren Sie das Leben, dann können Sie auch Ihre Patienten spüren."

Quelle: Presseaussendung Lebens.Med Zentrum Bad Erlach

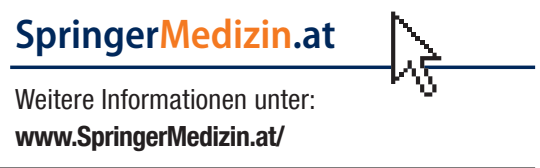

